\title{
High accuracy alignment facility for the receiver and transmitter of the BepiColombo Laser Altimeter
}

\author{
Sumita Chakraborty, ${ }^{1}$ Michael Affolter, ${ }^{1}$ Kurt Gunderson, ${ }^{1}$ Jakob Neubert, ${ }^{2}$ \\ Nicolas Thomas, ${ }^{1, *}$ Thomas Beck, ${ }^{1}$ Michael Gerber, ${ }^{1}$ Stefan Graf, ${ }^{1}$ \\ Daniele Piazza, ${ }^{1}$ Antoine Pommerol, ${ }^{1}$ Guillaume Roethlisberger, ${ }^{1}$ \\ and Karsten Seiferlin ${ }^{1}$ \\ ${ }^{1}$ Physikalisches Institut, Universität Bern, Sidlerstrasse 5, 3012 Bern, Switzerland \\ ${ }^{2}$ IB Neubert GmbH, Gass 1a, 8451 Kleinandelfingen, Switzerland \\ ${ }^{*}$ Corresponding author: nicolas.thomas@ @space.unibe.ch
}

Received 3 April 2012; revised 3 June 2012; accepted 4 June 2012;

posted 4 June 2012 (Doc. ID 165991); published 9 July 2012

\begin{abstract}
The accurate co-alignment of the transmitter to the receiver of the BepiColombo Laser Altimeter is a challenging task for which an original alignment concept had to be developed. We present here the design, construction and testing of a large collimator facility built to fulfill the tight alignment requirements. We describe in detail the solution found to attenuate the high energy of the instrument laser transmitter by an original beam splitting pentaprism group. We list the different steps of the calibration of the alignment facility and estimate the errors made at each of these steps. We finally prove that the current facility is ready for the alignment of the flight instrument. Its angular accuracy is $23 \mu \mathrm{rad}$. (C) 2012 Optical Society of America
\end{abstract}

OCIS codes: $\quad 120.6085,280.3400,120.1680$.

\section{Introduction}

In 2015, the European Space Agency's (ESA) BepiColombo mission will start its journey toward Mercury. On board, the BepiColombo Laser Altimeter (BELA), the first interplanetary laser altimeter developed in Europe, will produce a detailed global map of Mercury's topography. One of the major challenges in the construction of this instrument is the coalignment of its transmitter to its receiver. The restricted field of view (FOV) of the receiver requires an alignment accuracy of $60 \mu \mathrm{rad}$ and knowledge of the mounting error within $50 \mu \mathrm{rad}$.

In this paper, we present the alignment facility developed to achieve these objectives. In the following paragraphs, we provide an introduction to the BELA experiment, which outlines the requirements for the alignment system.

$1559-128 \mathrm{X} / 12 / 204907-09 \$ 15.00 / 0$

(C) 2012 Optical Society of America

\section{A. Laser Altimetry}

The topography of a planetary surface can be mapped remotely to high accuracy by using a laser altimeter. A laser onboard a spacecraft (typical altitude of a few hundreds $\mathrm{km}$ ) emits a pulsed beam toward the surface. The laser wavelength is usually in the near infrared (Nd:YAG at $1064 \mathrm{~nm}$ ) for reasons of efficiency. Some of the light is absorbed by the materials on the surface, but a fraction of the beam is scattered. Part of this reflected light (typically a few thousand photons per square meter) reaches a telescope that focuses the received light onto a detector. The "time of flight" between the emission of the outgoing pulse and the detection of the backscattered pulse is measured and converted into the distance between the spacecraft and the surface [1]. Precise knowledge of the spacecraft position is then required to retrieve the altitude values with a vertical resolution in the submeter range. It also permits the active measurement of the surface reflectance at zero phase angle (incidence $=$ emission direction) 
and provides insight into the meter-scale roughness of the surface from the shape of the reflected pulse.

Because of these advantages, this method has already been successfully used several times in the past to characterize the surfaces of planets (Mars Orbiter Laser Altimeter on Mars [2] and Mercury Laser Altimeter on Mercury [3]), the Moon (Lunar Orbiter Laser Altimeter "LOLA" [4]], Lunar Laser Ranging Instrument on Chandrayaan-1 [5], Laser ALTimeter on Kaguya [6], and the laser altimeter on Chang'e1 [7]), and asteroids (Near Earth Asteroid Rendezvous on Eros [8] and Hayabusa LIDAR on Itokawa [9]). The ESA's BepiColombo mission to Mercury will carry a laser altimeter system called BELA [10]. It is to be expected that in the future, laser altimeters will be a standard element of the scientific payload of most planetary missions to objects with low atmospheric opacities.

\section{B. Restrictions on the Field of View of Laser Altimeters}

The laser spot on the surface, of a finite size, is detected by an avalanche photodiode (APD) in the focal plane of a telescope. The APD subtends an angular width at the surface controlled by the telescope optics. This angular width must be large enough to encompass the laser spot, taking into account all alignment errors and distortions (both static and dynamic).

A band-pass filter is used in the detection system to extract spectrally the return laser pulse from the background radiation produced by sunlight reflected from the surface on the dayside of the planet. If the temperature of the instrument is not constant, the wavelength of the laser shifts with temperature. As a consequence, the width of the band-pass filter has to be increased, resulting in a larger level of background radiation.

To limit the increase in background, the FOV of the receiver has to be made as small as possible while maintaining (to a high probability) the laser spot within this FOV. The smaller the FOV, the tighter the requirements on the accuracy of the alignment between the transmitter laser (Tx) and the receiver telescope $(\mathrm{Rx})$. The stability of the alignment over one orbit also has to be guaranteed.

In this paper, we describe the concept of the facility designed and constructed at the University of Bern to align the Tx to the Rx of BELA and measure the misalignment between these two instrument components. In the next section, we explain in further quantitative detail the reasons why a tight alignment is required. In Section $\underline{3}$, we discuss different alignment concepts and explain the reasons for the choice of a large collimator system, now implemented in the "Star Simulator" facility. The original beam splitting/attenuation system required to handle the power of the emitter laser is presented in the Section 4 . The actual laboratory setup and its calibration are described in Section 5 and the final alignment procedure is presented in Section 6 .

\section{BELA}

\section{A. Instrument Description}

BELA's transmitter part is built under the responsibility of DLR Berlin and the receiver part under the responsibility of the University of Bern. The MaxPlanck-Institut für Sonnenforschung, the Instituto de Astrofísica de Andalucía, and multiple industrial partners are also involved in the project. A summary of the main instrument characteristics is shown in Table 1 and Fig. 1. A beam expander in front of the laser head box (LHB) broadens the laser beam and reduces its divergence. In that way, the spot size on the surface of Mercury is kept small $(60 \mathrm{~m}$ at a spacecraft height of $1000 \mathrm{~km}$ ). The reflected light will be collected by a $200 \mathrm{~mm}$ beryllium Ritchey-Chrétien Cassegrain telescope, which focuses the signal onto an APD detector. A reflective baffle in front of the telescope prevents solar straylight from reaching the detector. The on-board electronics then derives the time of flight and thus the distance between the spacecraft and the surface of the planet, as well as some geometric characteristics of the reflected pulse used to retrieve information on Mercury's surface. A complete and detailed description of the BELA instrument is provided by [10].

\section{B. Alignment Error Sources}

There are numerous reasons why the lines of sight (LoS) of Rx and Tx may be misaligned with respect to each other. The error budget (Table 2) was constructed as a combination of several contributions to the total error. The total misalignment must be lower than $225 \mu \mathrm{rad}$, to ensure that the laser spot at the surface of Mercury is within the FOV of a telescope. In addition to the initial mounting error, other sources of misalignment can exist. Slippage during launch and thermal flexure can occur, the latter being exacerbated by the strongly varying heat load at Mercury. Therefore we make further allocations for misalignment caused by thermal and mechanical movement of the $\mathrm{Tx}, \mathrm{Rx}$, and the mounting plate $(C, D$, and $E)$. It was necessary to separate these individual contributions for interface reasons (allocation of responsibility). The laser has a divergence

Table 1. BELA Characteristics

\begin{tabular}{ll}
\hline Laser type & $Q$-switched Nd:YAG \\
Pulse rate & $10 \mathrm{~Hz}$ \\
Pulse energy & $50 \mathrm{~mJ}$ \\
Beam diameter (after beam & $\leq 74 \mathrm{~mm}$ \\
expander) & \\
Beam divergence & $60 \mu \mathrm{rad}$ \\
Receiver telescope & Ritchey-Chrétien \\
& Cassegrain \\
Receiver focal length & $1250 \mathrm{~mm}$ \\
Receiver field of view & $450 \mu \mathrm{rad}$ \\
Primary mirror diameter & $200 \mathrm{~mm}$ \\
Detector type & Avalanche Photo-Diode \\
Detector size & $0.8 \mathrm{~mm}$ circular \\
\hline
\end{tabular}




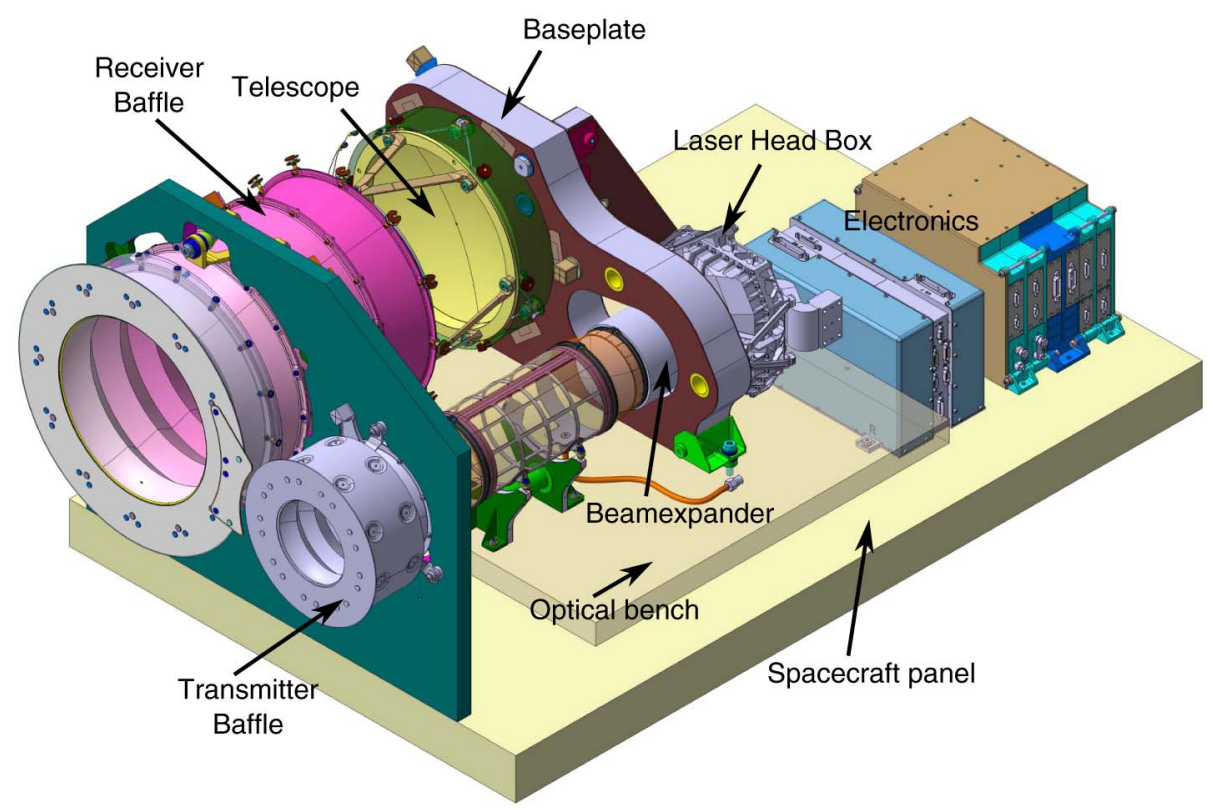

Fig. 1. (Color online) CAD/CAM of the BELA instrument.

$(G)$, which should be known and the spacecraft motion during the light travel time to and from the surface must be taken into account. This leads to an "aberration angle" between the direction in which the laser was emitted and the position where the $\mathrm{Rx}$ detects the return signal. The maximum "aberration angle" appears at periherm, when the spacecraft is closest to the planet. An additional allowance is made for image blur by the Rx. This is additive in the budget, but this is not strictly accurate since this will be superimposed upon the laser divergence. Depending on the nature of the sources, errors must be combined in a quadratic (independent random errors) or linear way (systematic errors).

The total misalignment error $(225 \mu \mathrm{m})$ and the estimates for other sources of misalignment provided in Table 2 constrain the accuracy of the initial alignment of the Tx and the $\mathrm{Rx}$ to about $80 \mu \mathrm{rad}$. To take into account the limitations and own error sources of the alignment facility, it was decided to split the

Table 2. Alignment Error Budget

\begin{tabular}{lc}
\hline Quantity & Allowed Error $[\mu \mathrm{rad}]$ \\
\hline Initial Rx-Tx misalignment $(A)$ & 60 \\
Knowledge of mounting error $(B)$ & 50 \\
Tx in flight misalignment $(C)$ & 49 \\
Rx in flight misalignment $(D)$ & 49 \\
Baseplate distortion $(E)$ & 83 \\
$F=\sqrt{A^{2}+B^{2}+C^{2}+D^{2}+E^{2}}$ & 133 \\
Laser divergence $(G)$ & 30 \\
Image blur $(H)$ & 36 \\
Point ahead angle $(I)$ & 18 \\
Minimum required Rx FOV radius & 217 \\
$(F+G+H+I)$ & \\
Margin & 8 \\
Total Rx FOV radius & 225 \\
\hline
\end{tabular}

requirement for the initial alignment in two parts: the actual mounting error of the Tx and $\mathrm{Rx}$ and the knowledge of this mounting error that directly depends on the resolution of the alignment facility. Initial estimates of the performances of the alignment facility at the beginning of its development lead to a plausible angular resolution of about $50 \mu \mathrm{rad}$. Because the actual mounting error and the knowledge of the mounting error are independent sources of errors, the combined error can be calculated from their quadratic sum. From the assumed angular resolution of $50 \mu \mathrm{rad}$ and from the allowed total initial misalignment of $80 \mu \mathrm{rad}$, a value of $60 \mu \mathrm{rad}$ for the actual mounting error was thus calculated.

\section{Alignment Concept}

Different approaches exist to align two optical elements to each other. The simplest one would be to use an autocollimator with an absolute accuracy of a few $\mu$ rad mounted on a translator. However, as our alignment system will also be used to perform a functional test of the BELA instrument, we need a setup where the laser signal can be measured and the telescope can be illuminated at the same time, which prevents the use of an autocollimator for alignment. Another option would be to use a retroreflector equipped with a partially reflecting coating to attenuate the BELA laser. The return signal could then be directly viewed by the receiver telescope. However, due to the start pulse processing, the return signal cannot be detected earlier than $300 \mu \mathrm{s}$. This would mean that the light has to travel about $45 \mathrm{~km}$ until it is reflected back on the telescope, which is not achievable in the laboratory.

We thus decided to use a large collimating system. This alignment setup is built around an off-axis 
parabolic mirror (OPM) with a focal length of $2.5 \mathrm{~m}$ and a light source positioned at its focal point creating a collimated beam. A sketch of the alignment setup is shown in Fig. 2 (Config. 3). The Rx and the Tx are mounted into the collimated beam and aligned to the focus of the OPM. In this way, they are also aligned to each other.

A collimator system was also used at Goddard Space Flight Center for the alignment of the Mercury Laser Altimeter (MLA) [11] of the MESSENGER mission to Mercury [12]. However, these two facilities differ by the angular alignment resolution they can achieve. Table 3 shows that the integration requirements for the $\bar{T} x-R x$ alignment of MLA were much looser than the one for BELA. MLA's alignment was actually performed in two steps. First, the transmitter and receiver were mounted and only roughly aligned on a common baseplate. The focal plane assembly with the detector was then adjusted with higher accuracy.

As a consequence of contractual requirements, it will not be possible to adjust the position of the detector after the BELA receiver has been assembled. The entire alignment procedure is thus restricted to the Tx-Rx alignment. This implies that BELA's Tx and $\mathrm{Rx}$ have to be aligned with a much higher accuracy during the integration on the common baseplate (Table 3).

The $\bar{R} x$-Tx co-alignment will be carried out in the "Star Simulator" laboratory. This name refers to the use of a large collimator system, simulating collimated light received from distant stars.
Table 3. Alignment Requirements for BELA and MLA [11] Integration

\begin{tabular}{lcc}
\hline & MLA & BELA \\
\hline Rx to Tx & $<2 \mathrm{mrad}$ & $60 \mu \mathrm{rad}$ \\
Rx to baseplate (IAC) & $<5 \mathrm{mrad}$ & $70 \mu \mathrm{rad}$ \\
\hline
\end{tabular}

\section{Attenuation Requirements and Design of the Pentaprism Group}

The BELA laser has a pulse energy of $50 \mathrm{~mJ}$ at a repetition rate of $10 \mathrm{~Hz}$ with a pulse length of 2.5-8 ns. The energy density of the focused laser spot will be over $8 \mathrm{~J} / \mathrm{cm}^{2}$, which exceeds by far the laser induced damage threshold of the CCD detector that is monitoring the refocused spot (Fig. 2). This CCD detector saturates at $20 \mu \mathrm{J} / \mathrm{cm}^{2}$. An attenuation system is therefore needed so that only a minute fraction $\left(2.5 \cdot 10^{-6}\right)$ of the incoming energy can reach the detector. This attenuator must always stay in the light path.

In addition to the BELA laser light (Tx beam in Fig. 2), the light coming from the light source at the focus of the OPM (diode laser beam in Fig. 2) also has to pass through the attenuator. This guarantees that the attenuator does not introduce any offset between the two elements to be aligned. As a response to these constraints, a concept with two pentaprisms with dielectric coatings that can withstand the high laser peak power was developed (Fig. 3). pentaprisms are more suitable than a coated flat plate, because the pentaprisms compensate for rotation errors and

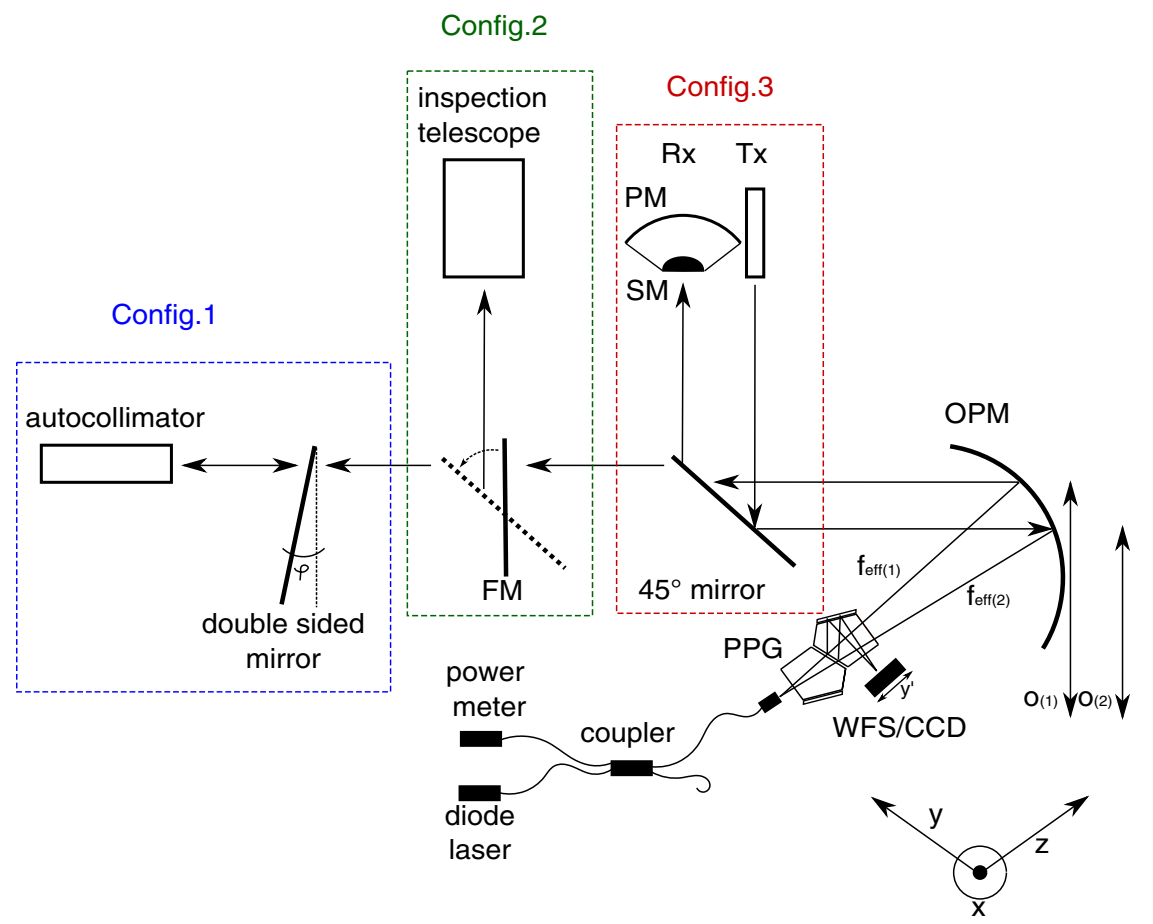

Fig. 2. (Color online) Sketch of the different setups used in the "Star Simulator" laboratory. The three dashed boxes indicate three different possible configurations while the rest of the setup is fixed. Configuration 1: Verification of the tilt angle measurement linearity. Configuration 2: Fiber alignment to the focus of the off-axis parabolic mirror (OPM). Configuration 3: Tx-Rx co-alignment. PM, primary mirror; SM, secondary mirror; PPG, pentaprism group; WFS, wavefront sensor; FM, folding mirror. 


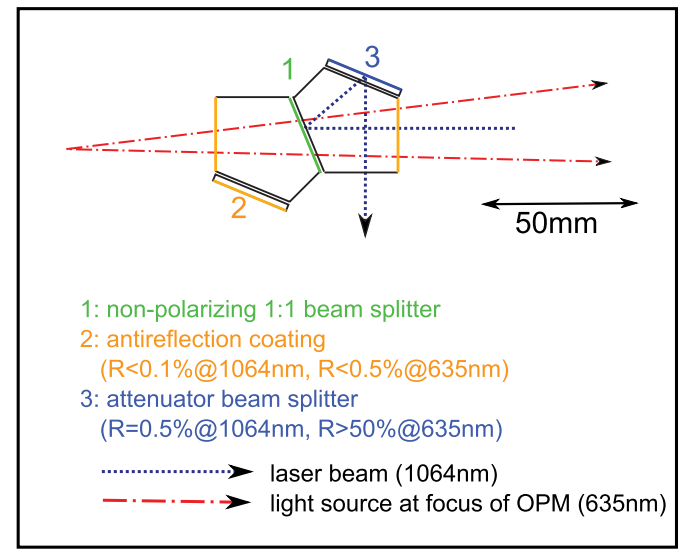

(a)

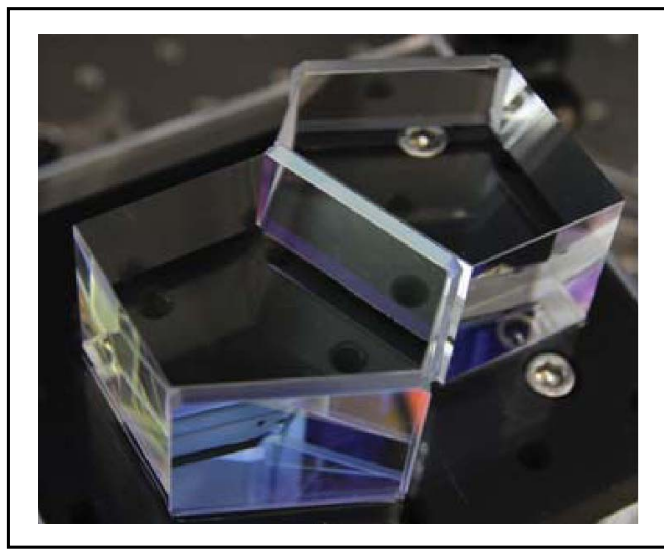

(b)

Fig. 3. (Color online) Sketch (a) and picture (b) of the pentaprism group used for attenuation and beam splitting.

thus reduce error sources. Between the two prisms is a $1: 1$ beam splitter coating [No. 1 in Fig. 3(a)]. The sides colored in yellow [No. 2 in Fig. 3(a)] have an antireflection coating $(R<0.1 \% @ 1064 \mathrm{~nm}$ and $R<$ $0.5 \%$ at $635 \mathrm{~nm})$. A small fraction $(0.5 \%)$ of the Tx beam is reflected from side No. 3 toward the direction of the CCD. The rest of the light is transmitted and directed into a beam trap. The light source in the focus of the OPM (diode laser beam in Fig. 2) has a wavelength of $635 \mathrm{~nm}$, with much less power than the BELA laser. The coating of surface No. 3 was therefore designed to reflect $50 \%$ of the light at $635 \mathrm{~nm}$. As a result, we obtain a good signal on the CCD both at $1064 \mathrm{~nm}$ and $635 \mathrm{~nm}$ and we are able to combine the beam splitting and the attenuation into a unique component. Layertec $\mathrm{GmbH}$ in Mellingen, Germany produced this custom-made pentaprism group.

\section{Setup Installation}

Before the actual BELA instrument alignment can be performed, the alignment setup has to be installed in the laboratory and properly characterized. The alignment setup installation can be summarized in the following steps:
A. Light source adjustment: The fiber light source is mounted into the focus of the OPM (Config. 2, Fig. 2)

B. Zero tilt calibration: A flat mirror (FM) is mounted in the collimated beam, and the light from the fiber light source is refocused onto the CCD. The position of the refocused spot is then measured when the mirror is not tilted (Config. 2, Fig. 2).

C. Tilt verification: To verify that a specific shift of the refocused spot on the CCD corresponds to the actual tilt of the mirror in the collimated beam (Config. 1, Fig. 2).

D. Instrument vertical mounting: BELA is assembled on a vertical mount and a $45^{\circ}$ mirror is used to direct the collimated light towards BELA (Fig. 7). It has thus to be verified that the $45^{\circ}$ mirror does not introduce any error in the light path.

\section{A. Light Source Adjustment}

\section{Optical Validation Calculation}

In order to make a reasonable estimation of the accuracy with which the fiber has to be aligned to the OPM, a Zemax [13] model of the setup was used. The figure errors of the mirrors used in the alignment setup were measured by the manufacturer and are reported in Table $\underline{4}$. These shape errors were also

Table 4. Equipment List of the Star Simulator Laboratory

\begin{tabular}{lll}
\hline Equipment & \multicolumn{1}{c}{ Provider } & Characteristics \\
\hline Off-Axis Parabolic Mirror (OPM) & Tydex & $\varnothing=510 \mathrm{~mm}$ \\
Autocollimator & Newport & Resolution $=0.1 \mu \mathrm{rad}$ \\
CCD & Melles Griot & Resolution $=1 \mu \mathrm{m}$ \\
Camera & Pixelfly PCO & Pixel size $=4.65 \times 4.65 \mu \mathrm{m}^{2}$ \\
Diode laser & Schafter + Kirchhoff & $635 \mathrm{~nm}, 5 \mathrm{~mW}$ \\
$45^{\circ}$ mirror & Berlinerglas & $\lambda 10 @ 635 \mathrm{~nm}$ \\
Double sided mirror & Halle & $\varnothing=102 \mathrm{~mm}, \lambda 5$ \\
Flat mirror (FM) & Halle & $\varnothing=206 \mathrm{~mm}, \lambda 20$ \\
Inspection telescope & Orion & Aperture $=250 \mathrm{~mm}, f=1^{\prime} 200 \mathrm{~mm}$ \\
Power meter & Oriel & Spectral range $=200-1^{\prime} 100 \mathrm{~nm}$ \\
PentaPrism Group (PPG) & Layertec & 2 pentaprisms $+2 \mathrm{plane} \mathrm{plates}$ \\
Linear and elevation stage & Micos & Repeatability: $\pm 0.2 \mu \mathrm{m}$ \\
Wavefront sensor (WFS) & Thorlabs & Resolution $=\lambda 50$ \\
\hline
\end{tabular}


implemented in the Zemax model. A worst-case assumption has been made by assuming that the wavefront error only consists in astigmatism with equal orientation (no compensation).

When the fiber is decentered by $0.5 \mathrm{~mm}$ perpendicular from the optical axis ( $x$ and $y$ in Fig. 2), the wavefront error increases by $30 \mathrm{~nm}$ and at a decenter of $1 \mathrm{~mm}$ by $70 \mathrm{~nm}$ (Fig. 4). As a diffraction-limited setup is required $(\lambda / 13=49 \mathrm{~nm} @ 635 \mathrm{~nm})$, the alignment accuracy has to be better than $\pm 1 \mathrm{~mm}$.

\section{Fiber Mounting}

One of the first steps in assembling the large collimating system is to mount the light source precisely to the focus of the OPM. The light source in the focus of the OPM is a diode laser connected to a fiber optic coupler via a monomode fiber (Fig. 2). One open end of the coupler is mounted at the focus of the OPM. The fiber adjustment can be separated into two steps:

- Finding the focal plane ( $z$ position) of the OPM with the inspection telescope (Config. 2 in Fig. 2 with FM tilted)

- Finding the focal point ( $x$ and $y$ positions) with the wavefront sensor (Config. 2 in Fig. $\underline{2}$ with FM vertical)

The fiber is first roughly positioned in the focal plane of the OPM. A $206 \mathrm{~mm} \mathrm{FM}$ is used to deflect the collimated beam into an inspection telescope focused to infinity (Config. 2 in Fig. 2). If the fiber is in the focal plane of the OPM, a diffraction limited spot will be visible in the focal plane of the inspection telescope. A PCO Pixelfly camera is used to monitor the image in the focal plane of the inspection telescope. We were able to find the focal plane with an accuracy of $100 \mu \mathrm{m}$.

In the next step, the FM is tilted perpendicular to the collimated beam (Config. 2 in Fig. 2 with FM vertical). The light is reflected back and refocused by the OPM onto the fiber. We monitor how much power is

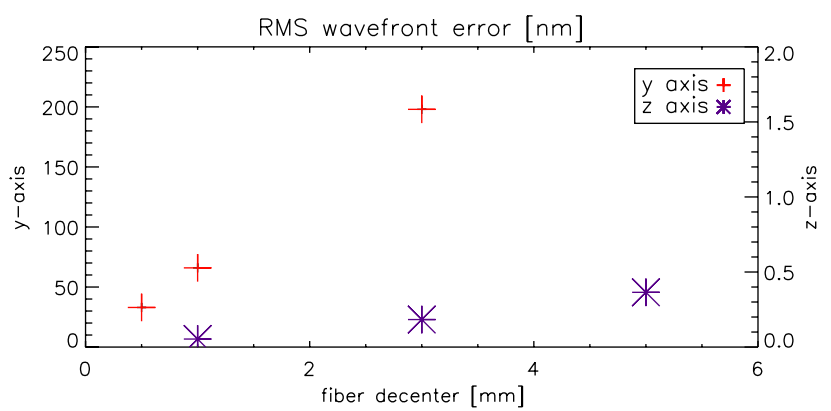

Fig. 4. (Color online) Wavefront error calculated with the Zemax model of the alignment setup. The wavefront error is plotted against the fiber decenter along the optical axis $z(*)$ and perpendicular to the optical axis $y(+)$. Tip, tilt, and defocus of the detector are not considered in the wavefront error calculation. A diffraction limited system requires wavefront error smaller than $49 \mathrm{~nm}$ for $\lambda=635 \mathrm{~nm}$. The resolution of the wavefront sensor (WFS) is $\lambda / 50=12.7 \mathrm{~nm}$. sent back through the fiber with a power meter, which is mounted at one end of the fiber optic coupler. The FM is tilted until the measured power is at maximum, at the optimal tilt angle. The power meter used for this measurement is an Oriel silicon detector from Newport (Table 4).

The backreflected and refocused light is split into several beams by the pentaprism group (Fig. 3). One fraction is observed with the Shack Hartmann wavefront sensor (WFS in Fig. 2). The WFS analyzes the incoming waveform by fitting zernike polynomials through the wavefront. The internal software is able to separate the total wavefront error into the individual contributions of tilt, defocus, astigmatism, and coma. Astigmatism and coma are mainly caused by displacement of the fiber in the $x-y$ plane (perpendicular to the optical axis of the OPM). While adjusting the position of the fiber, we observe the coefficients related to astigmatism and coma and find the position where the wavefront error is a minimum. The fiber is then in the focus of the OPM. Figure 5 shows that we were able to align the fiber within the required $< \pm 1 \mathrm{~mm}$.

\section{B. Zero Tilt Calibration}

The Zemax modeling shows that we are able to find the optimal position of the fiber with an accuracy of $\pm 1 \mathrm{~mm}$. Thus the remaining angular error on the test

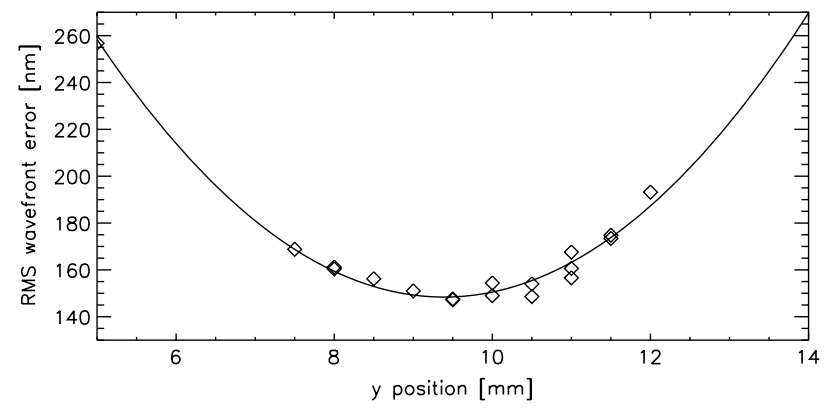

(a)

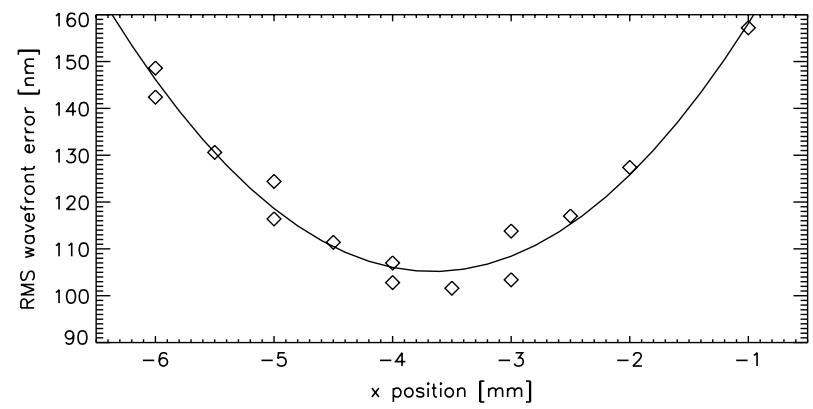

(b)

Fig. 5. Wavefront error was measured with a Shack Hartmann wavefront sensor while the fiber light source was adjusted to the focus of the off axis parabolic mirror. RMS wavefront error is plotted for different fiber positions, where $y$ (a) is the horizontal and $x$ (b) is the vertical displacement. First the $y$ axis was scanned to find the optimal horizontal position of the fiber and then the $x$ axis was scanned with the $y$ position set at optimum. The RMS error between the measured wavefront and the Zernike fit is $30 \mathrm{~nm}$. 
wavefront is $\arctan (2 \mathrm{~mm} / 2500 \mathrm{~mm})=0.8 \mathrm{mrad}$. This residual error can be compensated by the "calibration of zero tilt" with a CCD.

Once the right position of the fiber is found, the WFS is exchanged for a CCD detector, which is mounted in the focus of the refocused beam (Config. 2 in Fig. 2).

The $\overline{\mathrm{FM}}$ is adjusted in such a way that the maximum optical power is coupled back into the fiber. The spot centroid position is then measured on the CCD. This position corresponds to the "zero tilt" position that will be used as a reference for all future measurements. The measurement error of the CCD corresponds to an angular error of about $1.3 \mu \mathrm{rad}$. The smallest spot size we measured is $x=26 \mu \mathrm{m}$ and $y=31 \mu \mathrm{m}$. A diffraction limited spot diameter should be $19 \mu \mathrm{m}$. Our spot size is slightly larger because of atmospheric disturbances in the laboratory, caused by airflow used for contamination suppression. The spot position thus fluctuates and the image on the CCD gets blurred and appears larger. However, the centroid of the spot remains at the same position, which makes the definition of the zero tilt position accurate.

\section{Tilt Verification}

In the next step, we confirm that a specific tilt of a mirror in the collimated beam results in the expected shift of the spot centroid on the CCD. For that purpose, a double-sided mirror is mounted in the collimated beam (Config. 1 in Fig. 2). This mirror is then tilted by up to $10 \mathrm{mrad}$ relative to the zero tilt angle, and the shift of the spot on the CCD is monitored. The mirror tilt angle $\alpha$ is then calculated by

$$
\alpha=\frac{1}{2} \arctan \left(y^{\prime} / f_{\text {eff }}\right),
$$

where $y^{\prime}$ is the decenter from zero position on CCD and $f_{\text {eff }}$ is the effective focal length calculated by

$$
f_{\text {eff }}=\sqrt{f_{\mathrm{OPM}}^{2}+o^{2} / 2+o^{4} / 16 f_{\mathrm{OPM}}^{2}}
$$

where $f_{\mathrm{OPM}}=2500 \mathrm{~mm}$ is the focal length of the $\mathrm{OPM}$ and $o$ is the radial beam offset.

At the same time, the actual mirror tilt angle $\varphi$ is measured with an autocollimator from the other side. If $\alpha$ and $\varphi$ are the same for all tilts up to $10 \mathrm{mrad}$ from the zero tilt angle, we can measure accurately the tilt angles of the $\mathrm{Rx}$ and $\mathrm{Tx}$ with the CCD. Figure 6 shows the results of these tests. The mirror tilt angles derived from the CCD measurements are plotted against the angles measured by the autocollimator. For the vertical and horizontal tilt, the difference between the autocollimator and the CCD measurement slightly increases towards larger tilt angles. The maximum difference is $1.5 \mu \mathrm{rad}$. The CCD measurement error is $\pm 0.75 \mu \mathrm{rad}$ in the vertical and $\pm 1.1 \mu \mathrm{rad}$ in the horizontal direction.
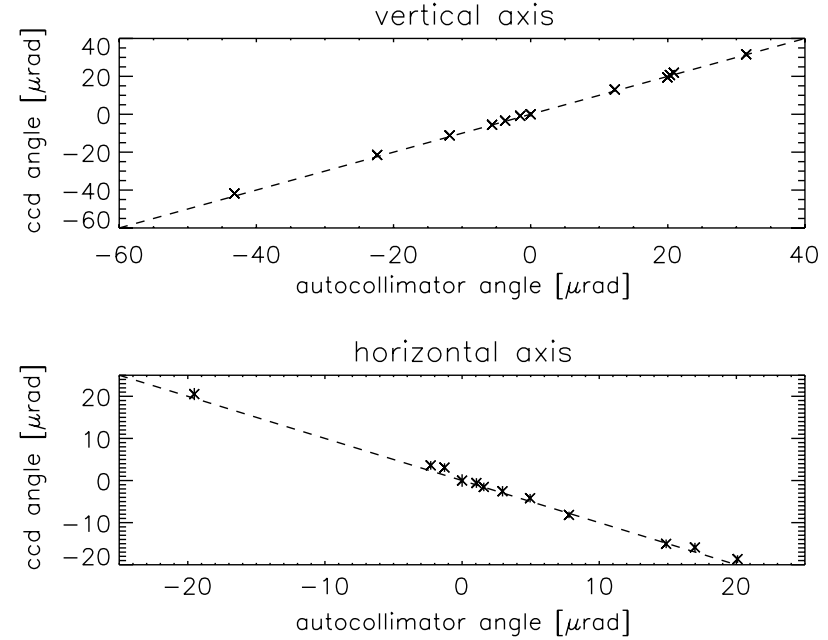

Fig. 6. Mirror tilt angle measurement from the autocollimator plotted against the CCD measurement for vertical (top) and horizontal (bottom) tilt. The measured points are shown as " $x$ " and the error on the CCD measurement is indicated by a vertical line through the " $x$." The errors on the autocollimator measurements are in the range of $0.1 \mu \mathrm{rad}$ and thus too small to appear on the plots. The dashed line represents the position of the ideal measurements.

\section{Vertical Mounting}

As BELA will be aligned on Earth, it will be unavoidable that gravity release effects on the different optical and mechanical elements will occur, which will cause misalignment. In order to limit gravity effects perpendicular to the optical axis, the Tx and the $\mathrm{Rx}$ will be oriented vertically on the alignment structure and facing downwards. A mirror tilted to $45^{\circ}\left(45^{\circ}\right.$ mirror in Fig. 2) is used to lead the collimated beam from the OPM into the BELA system (Fig. 7).

Before starting the alignment procedure, it has to be verified that the $45^{\circ}$ mirror does not significantly disturb the light path. Local irregularity on the $45^{\circ}$ mirror, for example, could introduce an additional tilt to the refocused light, which would cause an unnoticed misalignment.

To check the quality of the $45^{\circ}$ mirror, the FM, which was already used for the zero tilt calibration, was mounted on top of it, facing downward (in Fig. 7 the baseplate is replaced by the FM). A mask in front of the FM leaves two round openings separated by the same distance as between Tx and Rx. The refocused spots of these two beams have to overlay on the CCD, which is mounted in the focus of the OPM. Two spots appear when the CCD is defocused. The distance between these two spots is measured. The FM is then moved so that the entire collimated beam is scanned. At each position of the FM above the $45^{\circ}$ mirror, the distance between the two spots on the CCD should remain the same. Only in this case, the $45^{\circ}$ mirror will not introduce any error in the alignment procedure.

We measured the distance between the two refocused spots on the CCD for five different positions of the FM above the $45^{\circ}$ mirror. In each position, 




Fig. 7. (Color online) CAD sketch of the Tx and Rx mounted in vertical position facing downward. A folding mirror tilted to $45^{\circ}$ reflects the light toward the OPM. At the front is the light source on a $x y z$-table.

the spot position was measured about 25 times and the average value was calculated. The distance between the two points deviates by $\pm 6.8 \mu \mathrm{m}$ from position to position. This distance error caused by irregularities on the $45^{\circ}$ mirror on the CCD corresponds to an angular measurement error of $\pm 2.7 \mu \mathrm{rad}$. The standard deviation of the 25 measurements at one position is $\pm 13.4 \mu \mathrm{m}$, which corresponds to an angular error of $\pm 5.4 \mu \mathrm{rad}$. This measurement fluctuation was caused by noise on the CCD and the FM mirror mount, which tends to vibrate after the movement of the mirror.

\section{E. Accuracies and Error Budget}

Imperfections of the mirrors, fiber misalignment, and measurement errors on the WFS, the CCD, and the autocollimator are sources of systematic errors for the alignment measurement. An extensive summary of all errors in the alignment setup is provided in Table $\underline{5}$.

Some of the errors, but not all, can be compensated by calculation or calibration. The knowledge of the Rx-Tx alignment will thus have an uncertainty of $23 \mu \mathrm{rad}$ (RMS of the not compensated errors). The co-alignment can thus be achieved well within the required $50 \mu \mathrm{rad}$ alignment accuracy.

\section{Alignment Procedure}

\section{A. Telescope LoS Alignment to Zero Tilt Angle}

Once the setup is installed and calibrated, the actual Tx-Rx alignment of the BELA instrument can be performed (Config. 3, Fig. 2). In the first step of the alignment procedure, the $\mathrm{Rx}$ is mounted into the collimated beam and aligned to the focus of the OPM (the zero tilt position on the CCD). We will receive the Rx already fixed to the BasePlate (BP, Fig. 1), the Rx-Tx support structure, which will finally be fixed to the spacecraft. The BP with the telescope will be fixed on a frame on top of a vertical mount so that the instrument will look downwards (Fig. 7). In this way, the effects of Earth gravity will be aligned along the optical axis of the system, a configuration in which these gravity effects are less disturbing for instrument calibration. It is important to avoid applying mechanical stress on the BP, which could bend it and falsify the Rx-Tx alignment. The frame is therefore installed on top of spherical bearings. A mask is mounted in front of the $45^{\circ}$ mirror that only lets the collimated light reach the back of the secondary mirror (Fig. 7). The back of the Rx secondary mirror (SM in Fig. 2) is equipped with a flat mirror. In a previous step, the LoS of the Rx has been aligned with this mirror. In the step described here, it is only necessary to observe the flat mirror on the back of the Rx SM. When the refocused diode laser light that is reflected from the flat mirror on the back of the SM overlies with the outgoing diode laser light, it is aligned to OPM.

The whole baseplate is then tilted together with the frame with adjustment screws until the back

Table 5. Summary of Error Sources in the Alignment Setup for a Field Angle of $1 \mathrm{mrad}$

\begin{tabular}{lcc}
\hline Error Source & $\begin{array}{c}\text { Estimated value at } \\
1 \mathrm{mrad} \text { Field Angle }\end{array}$ & Remark \\
\hline Change of $f_{\text {eff }}$ of OPM & $4.464 \mu \mathrm{rad}$ & Not compensated \\
Change of $f_{\text {eff }}$ through misalignment & $6.43 \mu \mathrm{rad}$ & \\
and aberration & $800 \mu \mathrm{rad}$ & Compensated with zero tilt \\
Fiber alignment & $21 \mu \mathrm{rad}$ & Not compensated \\
Autocollimator & $1.1 \mu \mathrm{rad}$ & Not compensated \\
Autocollimator-CCD offset & $1.3 \mu \mathrm{rad}$ & Not compensated, CCD resolution $=0.4 \mu \mathrm{rad}$ \\
CCD fluctuation & $2.1 \mu \mathrm{rad}$ & Not compensated \\
WFS & $2.5 \mu \mathrm{rad}$ & Compensated with WFS calibration \\
Collimation optics & $5.4 \mu \mathrm{rad}$ & Not compensated \\
$45^{\circ}$ mirror imperfections & $4 \mu \mathrm{rad}$ & Not compensated \\
Difference LoS to optical axis & & \\
\hline
\end{tabular}


focused light is as close as possible to the zero tilt angle. The position of the refocused light $\left(x_{\mathrm{Rx}}, y_{\mathrm{Rx}}\right)$ is measured and the residual tilt error $\varphi_{\mathrm{Rx}}$ is calculated with Eq. (י),

$$
\varphi_{\mathrm{Rx}}=\arctan \left(\frac{1}{f_{\mathrm{eff}}} \cdot \sqrt{\left(x_{\mathrm{Rx}}-x_{0}\right)^{2}+\left(y_{\mathrm{Rx}}-y_{0}\right)^{2}}\right),
$$

where $\left(x_{0}, y_{0}\right)$ is the zero tilt position on the CCD and $f_{\text {eff }}$ is the effective focal length (Fig. 2) of the OPM.

\section{B. Laser Alignment to Telescope LoS}

In the final step, the LHB is mounted to the BP with screws and shims (Tx in Fig. 2). The mask in front of the laser is removed. Because the OPM would focus the BELA laser light onto the fiber exit, a protection mirror in front of the fiber is required. This protection mirror prevents the fiber from damage by deflecting the refocused light into a beam dump. The laser is then switched on and the spot centroid position is measured on the CCD. The LHB is mounted to the BP with three bipods arranged in an equilateral triangle. By adjusting the thickness of the shims of these bipods, the direction of the Tx beam is adjusted. The thickness of the shims can be changed by micrometers, which allows the tilting of the laser in $5 \mu \mathrm{rad}$ steps until the angle between Rx and Tx is as small as possible (and lower than $60 \mu \mathrm{rad}$ ).

\section{Conclusion and Future Work}

We have designed and constructed an original ground facility to align the transmitter to the receiver of BELA. By design, the BELA instrument requires a very accurate initial alignment $(<60 \mu \mathrm{rad})$ for which standard alignment concepts, like the ones used for LOLA and MLA integration, have to be modified. We have built the BELA alignment facility ("Star Simulator" laboratory) inside a class 100 clean room at the University of Bern. A light source is mounted at the focus of an OPM that acts as a large collimator. The Tx and receiver $\mathrm{Rx}$ are mounted into the collimated beam and are both aligned to the focus of the OPM. During the entire alignment procedure, the instrument is installed vertically so that the Earth gravity acts along the optical axis of the system. A newly developed pentaprism group is used to combine the laser attenuation and beam splitting in a single optical element.

We have proven by extensive testing of the equipment and optical modeling that the alignment setup has an accuracy of about $23 \mu \mathrm{rad}$. We have presented in detail the procedure to prepare the alignment setup and perform the alignment itself. The facility is now ready for the actual receiver-transmitter alignment of the flight model of BELA.

We acknowledge funding from the Swiss National Science Foundation (SNSF) under grant 200020_129932 and from the ESA PRODEX program under PRODEX Experiment Agreement C90191. We thank two anonymous reviewers for their constructive comments and Reinald Kallenbach for his assistance.

\section{References}

1. C. S. Gardner, "Ranging performance of satellite laser altimeters," IEEE Trans. Geosci. Remote Sens. 30, 1061-1072 (1992).

2. D. E. Smith, M. T. Zuber, S. C. Solomon, R. J. Phillips, J. W. Head, J. B. Garvin, W. B. Banerdt, D. O. Muhleman, G. H. Pettengill, G. A. Neumann, F. G. Lemoine, J. B. Abshire, O. Aharonson, D. Brown, S. A. Hauck, A. B. Ivanov, P. J. McGovern, H. J. Zwally, and T. C. Duxbury, "The global topography of Mars and implications for surface evolution," Science 284, 1495 (1999).

3. M. T. Zuber, D. E. Smith, S. C. Solomon, R. J. Phillips, S. J. Peale, J. W. Head, S. A. Hauck, R. L. McNutt, J. Oberst, G. A. Neumann, F. G. Lemoine, X. Sun, O. Barnouin-Jha, and J. K. Harmon, "Laser altimeter observations from MESSENGER's first Mercury flyby," Science 321, 77 (2008).

4. D. E. Smith, M. T. Zuber, G. A. Neumann, F. G. Lemoine, E. Mazarico, M. H. Torrence, J. F. McGarry, D. D. Rowlands, J. W. Head, T. H. Duxbury, O. Aharonson, P. G. Lucey, M. S. Robinson, O. S. Barnouin, J. F. Cavanaugh, X. Sun, P. Liiva, D. d. Mao, J. C. Smith, and A. E. Bartels, "Initial observations from the Lunar Orbiter Laser Altimeter (LOLA)," Geophys. Res. Lett. 37, L18204 (2010).

5. J. A. Kamalakar, K. V. S. Bhaskar, A. S. Laxmi Prasad, R. Ranjith, K. A. Lohar, R. Venketeswaran, and T. K. Alex, "Lunar ranging instrument for Chandrayaan-1,” J. Earth Sys. Sci. 114, 725-731.2005

6. H. Araki, S. Tazawa, H. Noda, Y. Ishihara, S. Goossens, S. Sasaki, N. Kawano, I. Kamiya, H. Otake, J. Oberst, and C. Shum, "Lunar global shape and polar topography derived from kaguya-lalt laser altimetry," Science 323, 897-900 (2009).

7. J. Wang, R. Shu, W. Chen, J. Jia, B. Wang, G. Huang, Y. Hu, and X. Hou, "Laser altimeter of CE-1 payloads system," Sci. China G: Phys. Astron. 53, 1914-1920 (2010)

8. M. T. Zuber, D. E. Smith, A. F. Cheng, J. B. Garvin, O. Aharonson, T. D. Cole, P. J. Dunn, Y. Guo, F. G. Lemoine, G. A. Neumann, D. D. Rowlands, and M. H. Torrence, "The shape of 433 Eros from near-shoemaker laser rangefinder," Science 289, 2097-2101 (2000).

9. O. S. Barnouin-Jha, A. F. Cheng, T. Mukai, S. Abe, N. Hirata, R. Nakamura, R. W. Gaskell, J. Saito, and B. E. Clark, "Smallscale topography of 25143 Itokawa from the Hayabusa laser altimeter," Icarus 198, 108-124 (2008).

10. N. Thomas, T. Spohn, J.-P. Barriot, W. Benz, G. Beutler, U. Christensen, V. Dehant, C. Fallnich, D. Giardini, O. Groussin, K. Gunderson, E. Hauber, M. Hilchenbach, L. Iess, P. Lamy, L.-M. Lara, P. Lognonné, J. J. Lopez-Moreno, H. Michaelis, J. Oberst, D. Resendes, J.-L. Reynaud, R. Rodrigo, S. Sasaki, K. Seiferlin, M. Wieczorek, and J. Whitby, "The BepiColombo Laser Altimeter (BELA): Concept and baseline design," Planet. Space Sci. 55, 1398-1413 (2007).

11. L. Ramos-Izquierdo, V. Stanley Scott, III, S. Schmidt, J. Britt, W. Mamakos, R. Trunzo, J. Cavanaugh, and R. Miller, "Optical system design and integration of the Mercury Laser Altimeter," Appl. Opt. 44, 1748-1760 (2005).

12. J. F. Cavanaugh, J. C. Smith, X. Sun, A. E. Bartels, L. Ramos-Izquierdo, D. J. Krebs, J. F. McGarry, R. Trunzo, A. M. Novo-Gradac, J. L. Britt, J. Karsh, R. B. Katz, A. T. Lukemire, R. Szymkiewicz, D. L. Berry, J. P. Swinski, G. A. Neumann, T. M. Zuber, and D. E. Smith, "The Mercury Laser Altimeter Instrument for the MESSENGER mission," Space Sci. Rev. 131, 451-479 (2007).

13. Zemax, "Optical design program user's guide” (2007). 